\title{
Operative Management of Neurogenic Bladder Dysfunction in Children: Ureterostomy
}

\author{
JAMES LISTER, R. C. M. COOK, „ and R. B. ZACHARY \\ From the Congenital Anomalies Research Unit, Department of Child Health, University of Sheffield, \\ and the Children's Hospital, Western Bank, Sheffield
}

In the child with a neurogenic bladder, diversion of the urinary stream may be indicated for the attainment of confident dryness and social acceptance in the incontinent child, or for the preservation of renal function.

For the first few years of life lack of bladder control is acceptable and life with nappies is expected (Smith, 1965; Nash, 1957; Carlson, 1966). In the child with anaesthetic buttocks and perineum, however, special care is necessary during these years for the avoidance of trophic ulceration, and steps must be taken to avoid urinary stagnation and to recognize and treat any urinary infection as soon as it occurs.

Regular timed bladder expression is generally recommended to avoid stagnation (Williams, 1956; Nash, 1957; Smith, 1965; Carlson, 1966), and some workers (e.g. Williams, 1960; Carlson, 1966) have been prepared to continue with this regimen until the age of 8 or 9 years or even older in the hope of achieving reasonable bladder control. The use of antibiotics or chemotherapy as prophylactic agents may be of some value in reducing the degree of renal damage due to recurrent infection (Lorber, Menneer, and Allott, 1968).

Provided the upper renal tract remains normal, bladder incontinence is not necessarily an indication for diversion of the urinary stream.

A boy with dribbling incontinence can readily be fitted with an appliance which will keep him dry, though even without urinary infection he may develop such severe ulcerative dermatitis in his anaesthetic scrotum and perineum that a diversion has to be made. A girl with dribbling incontinence cannot be fitted with a satisfactory appliance, but some girls (and boys too) can remain dry for two hours or more after bladder expression and we have taken this as an acceptable period of dryness, and thus such a child is not a candidate for diversion on the grounds of social acceptance.

Received June 13, 1968.

^Supported by a Grant from 'Action for the Crippled Child'.
Smith (1966) found such a high incidence of urinary infection after the age of 2 years that he considered persistence beyond that age with efforts at achieving bladder training and continence to be unjustifiable. All too often we have found persistent infection in children over the age of 2 , and this is frequently associated with severe upper urinary dilatation and renal insufficiency, so that urinary diversion has been a salvage operation which has perhaps slowed down the progressive destruction of renal tissue but has been too late either to allow reversal of the dilatation, or control of the chronic urinary infection.

Like others, therefore, we have felt increasingly conscious of the need for early assessment with a view to possible earlier operative procedures. Diversion on purely social grounds can presumably be undertaken at any time; but if one accepts that the renal insufficiency in children with spina bifida is due to progressive renal destruction as a result of infection of inadequately draining urine, then there is considerable urgency in achieving adequate drainage of the renal tract. Such drainage may sometimes be achieved by procedures to widen the bladder neck, but often it can only be achieved by diverting the urinary stream above the bladder.

Early assessment of the urinary tract in these children may involve undertaking radical procedures at a very early age, and we thought it of value to review our own experiences in the Paediatric Surgical Unit at the Sheffield Children's Hospital over four years, in order to assess the results of various procedures.

\section{Material}

During the period January 1963 to December 1966, 132 patients had surgical procedures designed to improve urinary drainage; ureterostomies are considered here, diversions through ileal conduits are described elsewhere (Cook, Lister, and Zachary, 1968).

In that period 52 ureterostomies were made in 31 patients with neurogenic bladder disease resulting 
from myelomeningocele or lipoma of the cauda equina. Their age and sex distribution is shown; most of the ureterostomies were made before the age of 5 years, and a significantly large proportion ( 9 out of $52-17 \%$ ) at less than 1 year (Fig. 1).

\section{Indications}

The general indications for supravesical urinary diversion have already been mentioned. Once it has been decided that the bladder must be abandoned, then we have usually chosen to undertake the simplest procedure and thus aimed, if possible, to make a ureterocutaneous spout, interposing a conduit of ileum or colon only if the ureter itself is either too short or too small in calibre to allow the placing of the spout in a situation where an effective collecting apparatus can be worn. The group of ureterostomies, therefore, is unlikely to include those children in whom diversion is recommended on social grounds alone because their ureters will be of normal calibre, but it does include many children in whom diversion is recommended on the grounds of upper urinary tract dilatation. It also includes a number of children in whom the ureterostomy has been made as a life-saving procedure, possibly intended to be only a temporary measure; examples of this are children who have had an anastomotic breakdown after a loop diversion, and children who have been in such severe renal insufficiency that a temporary drainage of the upper urinary tract had to be made in order to make them fit for the more traumatic procedure of making an ileal or sigmoid loop; this latter group of children may have such severe renal damage that the 'temporary' ureterostomy becomes a permanent one. A third type of temporary ureterostomy has been that used as a prognostic procedure; even with intravenous pyelography and isotope renography it is not always possible to assess the function of a grossly dilated kidney; implantation of the ureter from such a kidney into an ileal or sigmoid loop may mean that a non-functioning sac of infection is being left as a diverticulum while at the same time nephrectomy may mean the sacrifice of a valuable number of nephrons. Temporary drainage of the ureter will give a clear indication of the function of such a kidney so that a decision can be made as to whether it should be preserved or removed.

The actual indications for operation were varied, but the cases fall into fairly clearly defined groups,
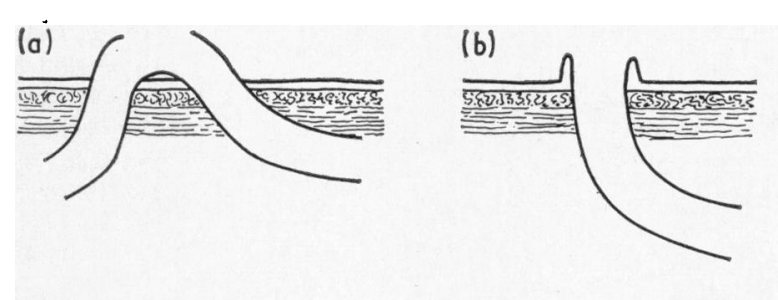

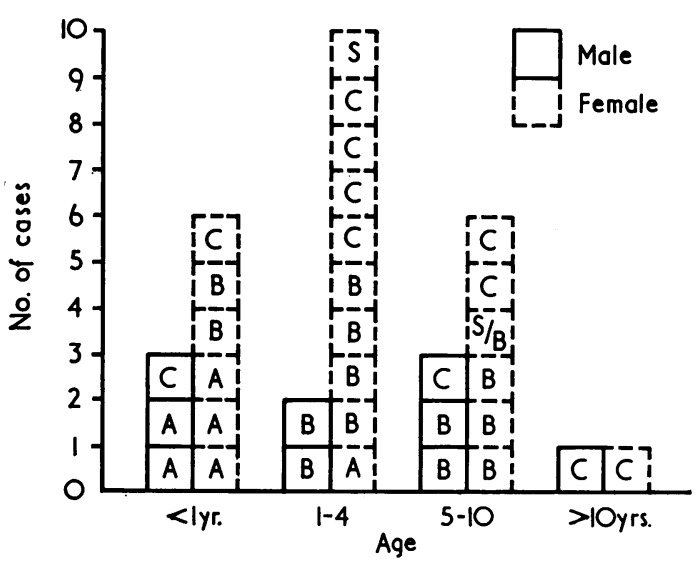

$A=$ Hydronephrosis with raised blood urea but no infection $B=$ Hydronephrosis with infection; normal blood urea C-Hydronephrosis with infection; raised blood urea $S=$ Normal ureters

Fig. 1.-Age, sex distribution, and condition of the urinary tract, in children treated by ureterostomy.

based on the presence or absence of infection and the level of the blood urea (Fig. 1).

Group A. Six of the children ( 2 boys and 4 girls) had hydronephrosis and hydroureters together with a raised blood urea, but uninfected urine; one of these was 16 months of age and the others were all less than 1 year. It is to be presumed that in these children the upper renal tract dilatation had developed in utero due to obstruction and a severe degree of renal damage was already present at birth.

Group B. Fourteen children had hydronephrosis and infection with a normal blood urea.

Group C. Ten children had a raised blood urea in addition to hydronephrosis and infection.

Group S. Two children had normal ureters brought to the skin surface after ischaemic necrosis and urinary leakage after an intestinal conduit had been constructed.

Operation. The exact form of operation was again a variable feature, but 3 general types have been made (Fig. 2).

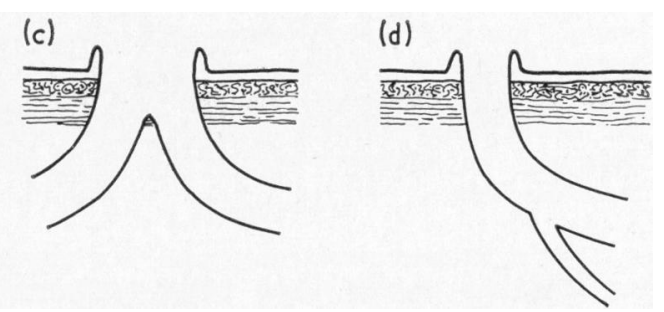

FIG. 2.-Diagrammatic representation of types of operation (a) Loop ureterostomy; (b) single terminal ureterostomy; (c) double-barrelled terminal ureterostomy: and (d) uretero-ureterostomy. 


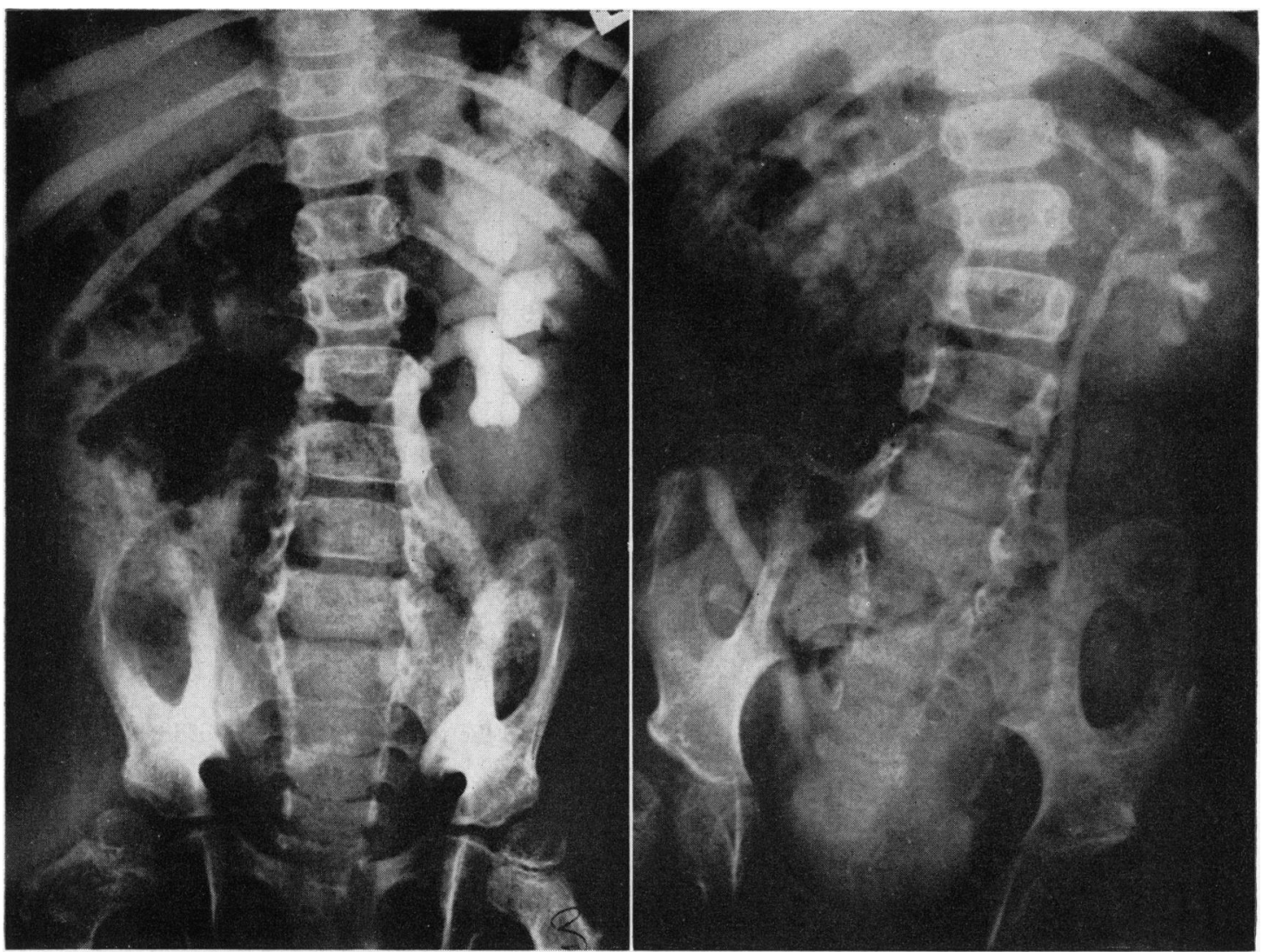

Fig. 3a.-Pre-operative pyelogram showing left hydronephrosis and no function on right side. b.-Intravenous pyelogram after right loop ureterostomy. Some function shown on right; considerable improvement on left. Left ureter draining retrograde from bladder to right ureterostomy. Note distal right ureter outlined by opaque medium refluxing from bladder.

(1) Loop ureterostomy. By an extraperitoneal approach, the ureter is exteriorized over a skin bridge in the iliac fossa and an opening made in it. Where there is a vesico-ureteric reflux on the side operated on, the resulting double stoma may drain the urine from both kidneys, none being passed per urethram. Such a unilateral procedure may result in considerable reduction of bilateral hydronephrosis (Fig. 3).

(2) Terminal ureterostomy. A single ureter is brought out at a site of election with respect to the appliance and the end 'fliped'* to form a short $(1 \mathrm{~cm}$.) spout. If the degree of dilatation and tortuosity of the ureters allow, both are brought out in the midline (following an extraperitoneal route) as a doublebarrelled stoma.

(3) Uretero-ureterostomy was used in a number of instances, the smaller ureter being anastomosed at

^For the benefit of the uninitiated Sassenach, 'flipe' is what the Scot has to do to his sock before he can put it on. Ed. its end to the side of a dilated one that was then used to form the stoma.

Complications. Though technically this has usually been a fairly simple and short operation, the general condition of the child has often been poor-this of course being one of the indications for the operation. In four cases renal failure was exacerbated. In 2 other children uretero-ureterostomy anastomoses have leaked causing cellulitis (or in one instance peritonitis). The terminal exteriorized portion of the ureter has undergone ischaemic necrosis in two instances and in two others the kidney has failed to drain because kinks had been left in the ureter, demanding revision. Infection has often been exacerbated, or superinfection with resistant Gram-negative organisms has followed antibiotic treatment. Wounds have been slow to heal. The effective fitting of appliances has been difficult except in the midline double-barrelled stomata.

\section{Results}

Mortality. 7 out of 31 children have since 
died (23\%). A 6-week-old girl (in Group A) with gross hydronephrosis of a solitary kidney and a raised blood urea had a loop ureterostomy made, but died on the 4th post-operative day.

A 6-week-old girl (in Group C) was found to have bilateral hydronephrosis and a raised blood urea and the urine was already infected. Bilateral loop ureterostomies were made. She remained fairly well for 7 months but then died of renal failure.

A 5-week-old girl (Group A) had bilateral terminal ureterostomies because of bilateral hydronephrosis and a raised blood urea. The urine was uninfected. She died of ventriculitis in an untreated hydrocephalus at the age of 7 weeks.

A 3-month-old girl with bilateral double kidneys and ureters had moderate hydronephrosis in three renal elements, and gross hydronephrosis in the fourth. The blood urea was within normal limits, but the urine was infected (Group B). The urine was diverted through a series of uretero-ureterostomies but after operation there was urine leakage, wound dehiscence, and renal failure from which the child died.

A 7-month-old boy was found to have a persistently raised blood urea. A pyelogram showed bilateral hydronephrosis and hydroureters. The urine was uninfected (Group A). Bilateral terminal ureterostomies were made and clinical improvement secured. He died at home one year later and the cause of death was not determined.

A girl of 13 months had bilateral terminal ureterostomies made for bilateral hydronephrosis, urinary infection, and a high blood urea. Irreversible renal failure occurred after operation.

A 4 $\frac{1}{2}$-year-old girl had a double-barrelled terminal ureterostomy made for hydronephrosis, infection, and a raised blood urea (Group C). She recovered well but defaulted in the follow-up. She died of uraemia 7 months after operation, the stoma of the ureterostomies being very stenosed.

The majority of these cases had ureterostomies made because of failing renal function, and those under 1 year, particularly those of 3 months and under, had diversions performed very largely as a salvage procedure. Of the 7 children who died, only 1 was over 13 months at the time of operation. It seems likely that this was the only death that could have been avoided: but post-operative follow-up had failed and she clearly required a further procedure to maintain her urinary drainage.

Survivors. Fig. 4 shows the present state of the kidneys and ureters in relation to their preoperative calibre.

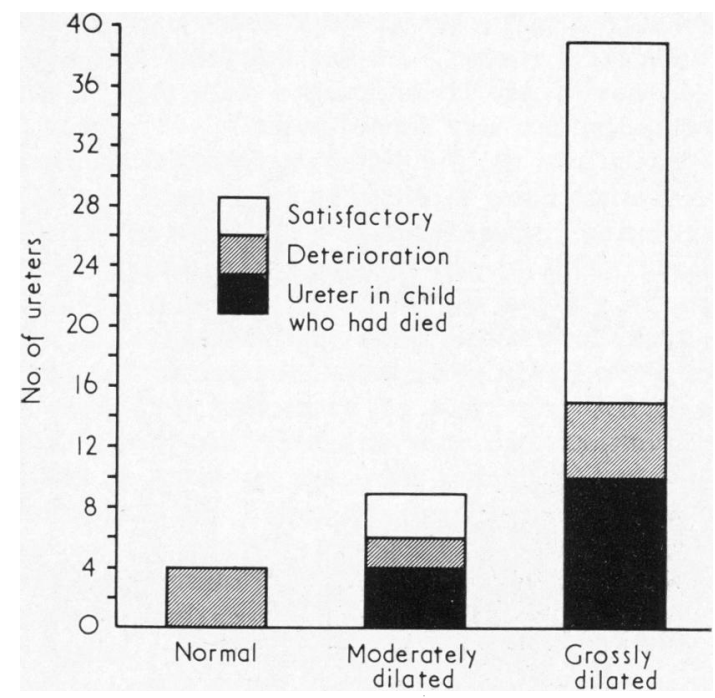

FIG. 4.-The results of ureterostomy in relation to the pre-operative calibre of the ureter.

Fig. 5 shows the results in relation to the 4 pre-operative groups (Groups S, A, B, and C); one patient appears twice in the 'satisfactory' column in Fig. 5, since ureterostomies were made at separate times-one in Group $A$ and one in Group B.

Both the children (Group S) with no upper

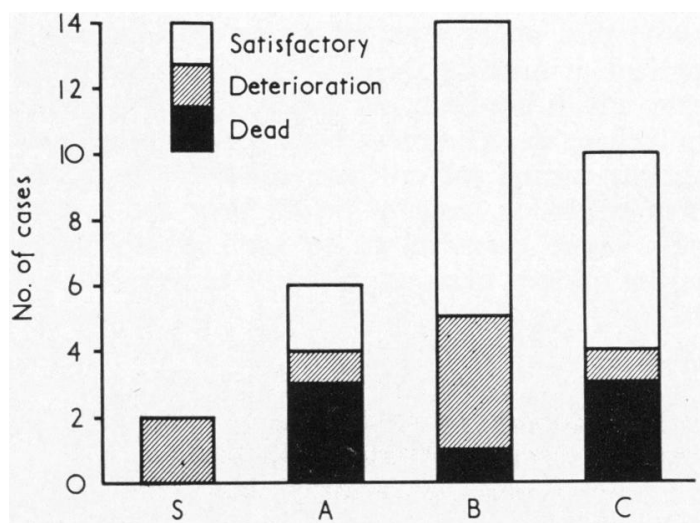

$A=$ Hydronephrosis with raised blood urea but no infection $B=$ Hydronephrosis with infection; normal blood urea

$\mathrm{C}=$ Hydronephrosis with infection; raised blood urea $S=$ Normal ureters

FIG. 5.-Results of individual children in relation to pre-operative grouping (one case appears twice, having a left ureterostomy at 8 years and a right at 10 years). 
renal tract pathology (as defined in the gross terms used here) required further operative procedures and could only be maintained with their ureterostomies for a very limited period.

Only 3 of the 6 children in Group A survived, 2 of them being accepted as satisfactory, the third requiring revision because of stenosis at the skin level. This group of children in fact forms a special group of very young children most of whom already have considerable renal damage at the time of birth. In Groups B and C the mortality is lower and the proportion of successful cases rises to 15 out of 24 ; understandably the mortality in Group C is higher than that in Group B, but in only 1 of the survivors of Group C did the ureterostomy prove unsatisfactory, whereas it was unsatisfactory in 4 of the Group B survivors. This is largely explained by the fact that the proportion of grossly dilated ureters was lower in Group B than in Group C, and Fig. 4 shows that, in terms of calibre, grossly dilated ureters have a greater success rate, but that the price of this success is the amount of renal damage acquired due to back pressure and infection while the ureter was becoming dilated.

\section{Discussion}

The indications for supravesical diversion of the urinary stream are so varied that differences of opinion in the literature as to the age of operation have arisen largely because of lack of clear definition of these indications.

It has been well shown by others (Eckstein, 1965) that upper renal dilatation may already be present at birth in the child with myelomeningocele, and it has been our routine for several years to include an intravenous pyelogram in the investigations carried out on these children during their first admission, usually before they are 4 weeks old. Those cases shown to have grossly dilated upper urinary tracts at this very early age usually fall into our Group A category, with evidence of renal failure but usually without severe infection as yet. These children require urgent drainage of the urinary tract, and as in other neonatal urinary outflow obstructions, bladder drainage will not always satisfactorily drain the tortuous dilated ureters. Loop ureterostomy in these children is likely to be the simplest, safest, and most effective form of treatment; not infrequently the gross hydronephrosis is unilateral, and the contralateral relatively undilated ureter and renal pelvis seems to be protected by the 'safety valve' bladder pressure release of the dilated side. A loop ureterostomy on the dilated side only preserves this 'safety valve' by allowing reflux to continue up the more dilated ureter. The parents of the child whose $x$-rays are shown in Fig. 3 were convinced that a complete urinary diversion had been made, because after a loop ureterostomy had been made on one side only the child stopped passing any urine per urethram, both kidneys draining by the loop ureterostomy.

In older children the indications for diversion will be combinations of incontinence, obstruction, and infection. Incontinence alone will demand the interposition of a loop of ileum or colon between ureter and skin, because in our experience the ureter of normal length and calibre cannot be brought to the surface at a site suitable for the wearing of an effective collecting apparatus, and all the kidneys drained by ureterostomies of normal calibre ureters showed deterioration at follow-up. Obstruction is an indication for diversion in many conditions other than neurogenic bladder disorders (Rickham, 1964), but in neurological disorders is in danger of being overlooked when it brings apparent 'control' to the incontinent (Smith, 1966), as the child may remain dry for lengthening periods. There is general agreement that obstruction is an indication for diversion at any age, but most writers are not specific about what they consider constitutes obstruction, other than the grosser manifestations of hydroureter and hydronephrosis.

The amount of residual urine is taken as one criterion of obstruction, but the permissible maximum allowed is not defined. Smith (1966) takes persistent or recurrent infection as evidence of obstruction, and unhesitatingly recommends diversion in these cases unless the infection is readily controlled by drugs.

There seems little doubt that where there is obstruction as evidenced by hydronephrosis and hydroureters, with or without infection, operative intervention is essential. Y-V Plasty or transurethral resection offers help in the mild cases. The grosser degrees of hydroureter are best managed by ureterostomy, not only because such dilated ureters form excellent stomata and will drain readily, but also because the clinical condition will preclude the more major operative procedures of intestinal conduit construction. In the past the presence of one normal or only mildly dilated ureter was taken to indicate the need for diversion through an intestinal conduit, but it is now well established that interureteric anastomoses can function well, and the smaller ureter can be implanted into the larger, which itself forms the stoma (Madden, Tan, and McCann, 1967). 

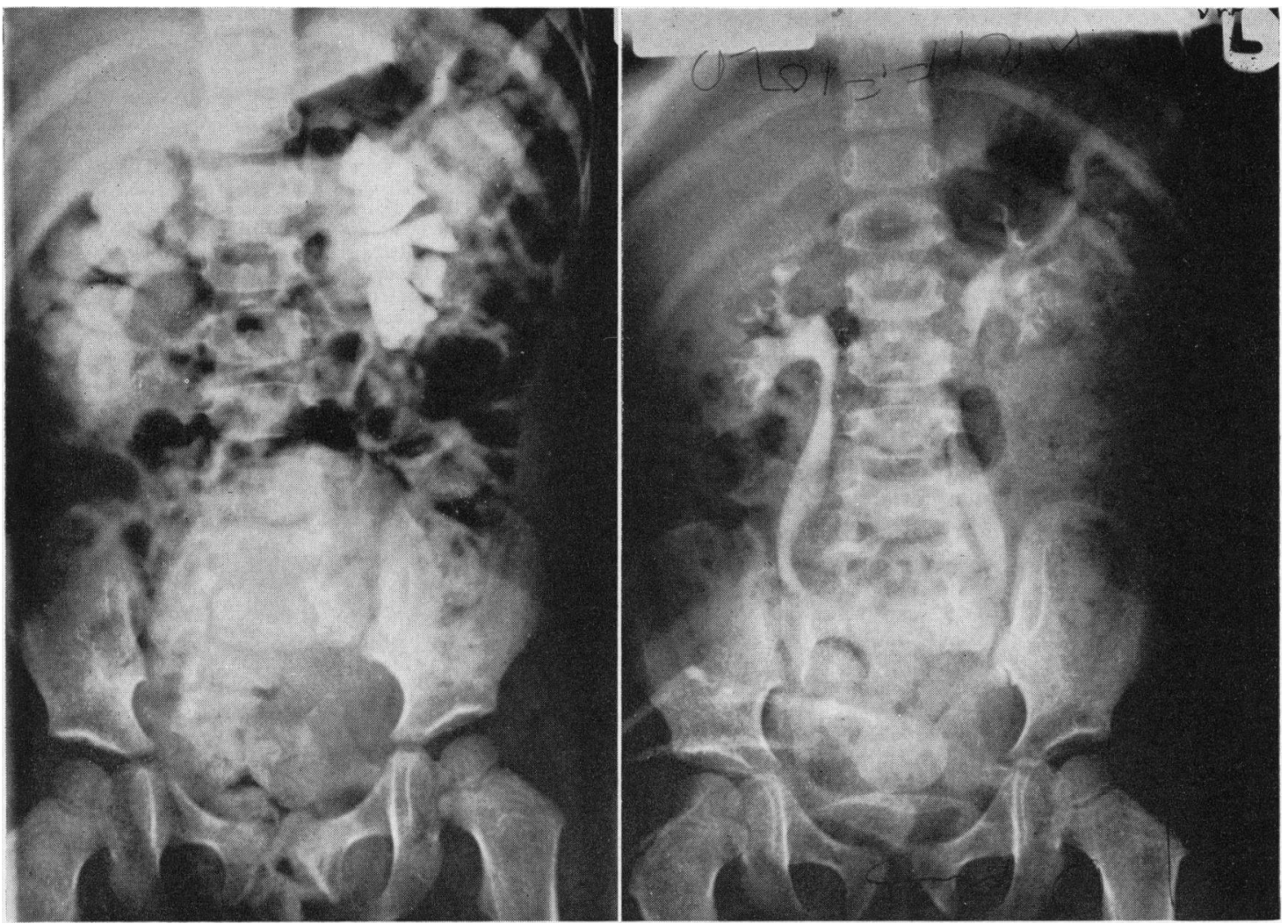

FIG. 6. a-Bilateral hydronephrosis. b-Recovery of both renal pelves following double-barrelled suprapubic ureterostomy.

Provided there has not been prolonged severe recurrent infection, and hydronephrosis is not gross, then the dilated kidney will usually recover once adequate drainage is achieved by ureterostomy (Fig. 6). However, the follow-up time in this series has been only from 1 to 5 years, and time may show further deterioration. One cannot deliberately delay operation and allow upper urinary tract dilatation in order to have a ureter of a suitable calibre to form a spout, since this undoubtedly will involve further renal damage. The aim of treatment in the neurogenic bladder must be the protection of the upper urinary tract; and thus urinary diversion should ideally be undertaken before there is marked upper urinary tract dilatation, at a stage when the calibre and length of the ureter is insufficient to allow the formation of a satisfactory cutaneous ureterostomy. Ureterostomy for neurogenic bladder, therefore, is indicated mainly in those children whose kidneys are already damaged and if the need for diversion is recognized at the proper time, then the procedure of choice will be an intestinal conduit; but nevertheless ureterostomy is a valuable second choice operation.

\section{Summary}

Experience in 52 ureterostomies carried out in 31 patients suffering from myelomeningocele is reviewed. 7 of these children died mainly from persistence of renal insufficiency but in one case there was progression of that insufficiency. Of 24 survivors, 7 have required further procedures, but $17(52 \%$ of total) have not deteriorated or have actually improved.

While it does not appear to have a place in urinary diversion when both ureters are of normal calibre, ureterostomy is particularly indicated in three situations in the child with a neurogenic bladder: (1) As a definitive procedure in the child with at least one dilated ureter in whom supravesical urinary diversion is required. (2) As an emergency measure, possibly temporary, a single loop ureterostomy in a unilateral hydroureter with 
bladder outflow obstruction; protecting the contralateral kidney by allowing reflux up and out of the dilated ureter, at the same time as preserving the dilated kidney. (3) As a temporary method of assessing function in the seriously deficient hydronephrotic kidney.

\section{REFERENCES}

Carlson, H. E. (1966). Urologic problems in meningornyelocele. 7. Urol. (Baltimore), 95, 245.

Cook, R. C. M., Lister, J., and Zachary, R. B. (1968). Operative management of the neurogenic bladder in children: diversion through intestinal conduits. Surgery, 63, 825.

Eckstein, H. B. (1965). Urinary diversion in children. Develop. Med. Child Neurol., 7, 167.
Lurber, J., Menneer, P. C., and Allott, D. C. (1968). An investigation into prophylactic treatment of urinary tract infections in infants born with spina bifida cystica. Develop. Med. Child Neurol., Suppl. 5, 30.

Madden, J. L., Tan, P. Y., and McCann, W. J. (1967). An experimental and clinical study of cross ureteroureterostomy. Surg. Gynec. Obstet., 124, 483.

Nash, D. F. E. (1957). Congenital spinal palsy. The management of incontinence. Ann. roy. Coll. Surg. Engl., 20, 349.

Rickham, P. P. (1964). Permanent urinary diversion in childhood. ibid., 35, 84.

Smith, E. D. (1965). Spina Bifida and the Total Care of Myelomeningocele. C. C. Thomas, Springfield, Illinois.

- (1966). Management of the urinary tract in spinal myelomeningocele: the case for early surgical diversion of urine. Aust. pediat. F., 2, 27.

Williams, D. I. (1956). The congenital neurogenic bladder. Proc. roy. Soc. Med., 49, 896.

- (1960). Management of the bladder in congenital spinal cord lesions. ibid., 53, 272. 